05

\title{
Эффект спинового насоса в системе ферромагнитный изолятор/нормальный металл: простая квантово-механическая модель
}

\author{
(C) E.A. Караштин ${ }^{1,2}$ \\ ${ }^{1}$ Институт фризики микроструктур РАН, \\ Нижний Новгород, Россия \\ ${ }^{2}$ Нижегородский государственный университет им. Н.И. Лобачевского, \\ Нижний Новгород, Россия \\ E-mail: eugenk@ipmras.ru
}

Поступила в Редакцию 15 апреля 2019 г.

В окончательной редакции 22 апреля 2019 г.

Принята к публикации 24 апреля 2019 г.

\begin{abstract}
Построена простая квантово-механическая модель эффекта спинового насоса при возбуждении ферромагнитного резонанса в магнитном диэлектрике, который имеет плоскую границу с немагнитным металлом. Модель основана на решении уравнения Шредингера для электронов проводимости в металле. Показано, что отражение электронов от изолятора с осциллирующей намагниченностью с процессом переворота спина приводит к появлению как постоянного, так и переменного (осциллирующего с частотой возбуждающего резонанс СВЧ-поля) потока спина из ферромагнетика в изолятор. При этом эффект постоянного тока мал по сравнению с эффектом переменного тока; в роли малого параметра выступает отношение обменной константы ферромагнетика к величине потенциального барьера между металлом и диэлектриком (больше энергии Ферми). Полученный результат согласуется с известными экспериментальными данными. Построенная модель дает простую и наглядную картину возникновения явления и позволяет проводить анализ зависимости эффекта от параметров исследуемой системы.
\end{abstract}

Ключевые слова: спиновый нанос, ферромагнитный резонанс, обменное взаимодействие, $s-d$ модель, спиновый ток.

DOI: $10.21883 /$ FTT.2019.09.48102.05N

\section{1. Введение}

Явление спинового насоса заключается в возникновении потока спина из ферромагнетика в граничащий с ним металл при возбуждении в магнитном материале ферромагнитного резонанса (ФМР). Данный эффект был впервые предложен в 2002г. [1,2] и с тех пор довольно много исследовался как теоретически, так и экспериментально [3]. В первоначальном варианте с этим эффектом связывалось уширение линии поглощения СВЧ-излучения ферромагнетиком при ферромагнитном резонансе (ФМР). Позже было показано, что за счет обратного спинового эффекта Холла [4] в результате эффекта спинового насоса в среде, в которую происходит накачка спина, возникает разность потенциалов. Таким образом, стала возможна регистрация как самого эффекта, так и ферромагнитного резонанса электрическим методом [5]. На результатах таких измерений обычно основан метод определения угла спинового эффекта Холла для разных проводников [6].

С симметрийной точки зрения спиновый ток $J_{i j}$, текущий из ферромагнетика с постоянной компонентой намагниченности $\mathbf{M}_{0}$ и осциллирующей намагниченно- стью $\mathbf{m}(t)$, может быть записан в виде

$$
J_{i j}=A_{1} \frac{d m_{j}}{d t} n_{i}+A_{2}\left[\left(\mathbf{M}_{0}+\mathbf{m}\right) \times \frac{d \mathbf{m}}{d t}\right]_{j} n_{i},
$$

где $\mathbf{n}$ - вектор нормали к границе ферромагнетика, в котором возбуждается ФМР, и нормального металла. Второе слагаемое формулы (1) дает ненулевое среднее значение при усреднении по периоду СВЧ-колебаний намагниченности. Именно оно описывает классический постоянный спиновый ток, возникающий при эффекте спинового насоса, и связанную с ним разность потенциалов. Первое же слагаемое формулы (1), очевидно, исчезает при усреднении. Оно описывает переменный спиновый ток, который на один-два порядка больше постоянного, но тем не менее был экспериментально зарегистрирован относительно недавно [7].

Эффект спинового насоса возникает как в случае системы ферромагнитный металл/немагнитный металл, так и для системы ферромагнитный диэлектрик/немагнитный металл. В первом случае он теоретически обычно получается в приближении туннельного гамильтониана [1]. Во втором же возникновение потока спина связывают с проникновением электронов проводимости на небольшую глубину в изолятор [8]. В рамках настоящей работы построена простая модель 
для случая ферромагнитный диэлектрик/немагнитный металл, основанная на квантово-механическом описании электронов проводимости в металле. Получен как постоянный, так и переменный эффект спинового насоса. Особенность ферромагнитного диэлектрика в данном случае заключается в том, что его потенциал $U$ больше величины энергии Ферми в соседствующем немагнитном металле. Процессы рассеяния на границе с переворотом спина здесь появляются в результате учета граничных условий, а проникновение волновой функции электронов проводимости в изолятор и спин-флип за счет этого получаются естественным образом. Построенная модель позволяет проводить простой анализ зависимости эффекта от параметров системы, таких, как амплитуда осцилляций намагниченности и величина потенциала в ферромагнитном диэлектрике.

\section{2. Теоретическая модель}

Гамильтониан электронов в ферромагнетике в записывается в рамках $s-d$ модели Вонсовского [9] и имеет вид

$$
\hat{H}=-\frac{\hbar^{2}}{2 m_{e}} \Delta+J(\hat{\sigma}, \mathbf{M}(t))+U,
$$

где намагниченность определяется выражением

$$
\mathbf{M}(t)=\left(m \cos \Omega t, m \sin \Omega t, \sqrt{1-m^{2}}\right),
$$

при этом потенциал $U$ больше энергии Ферми $\varepsilon_{F}$ в металле, находящемся рядом. Волновые функции электронов в такой среде могут быть найдены точно. Для дальнейшего построения теории мы предполагаем малость величины энергии СВЧ-кванта по сравнению с обменной энергией: $\hbar \Omega \ll J$ (такое условие выполняется для известных ферромагнетиков), а также малость обменной энергии по сравнению с энергией Ферми: $J \ll \varepsilon_{F}$. В то же время, предположения о малости амплитуды колебаний намагниченности $m$ не требуется.

Решение нестационарного уравнения Шредингера с гамильтонианом (2) имеет вид

$$
\psi_{ \pm}^{F}=\exp \left(-i \frac{\varepsilon_{ \pm}}{\hbar} t+i \mathbf{k r}\right)\left(\begin{array}{c}
a_{ \pm} e^{-i \Omega t} \\
b_{ \pm} e^{i \Omega t}
\end{array}\right)
$$

где энергия и коэффициенты спинора в линейном приближении по параметру адиабатичности $\beta=\frac{\hbar \Omega}{2 J}$ имеют вид

$$
\begin{gathered}
\varepsilon_{p m}=\frac{\hbar^{2} \mathbf{k}^{2}}{2 m_{e}} \pm \sqrt{\left(\frac{\hbar \Omega}{2}-\sqrt{1-m^{2}}\right)^{2}+J^{2} m^{2}} \\
\approx \frac{\hbar^{2} \mathbf{k}^{2}}{2 m_{e}} \pm J\left(1-\beta \sqrt{1-m^{2}}\right) \\
a_{+}=-b_{-}
\end{gathered}
$$

$$
a_{-}=-b_{+} \approx \frac{m}{\sqrt{2\left(1+\sqrt{1-m^{2}}\right)}}\left(1+\frac{\beta}{2}\left(1-\sqrt{1-m^{2}}\right)\right) .
$$

Рассматривая данное решение, заметим, во-первых, что хотя $\varepsilon_{ \pm}$содержит линейную по параметру адиабатичности поправку, собственные значения $\Psi_{ \pm}^{+} \hat{H} \Psi_{ \pm}$ гамильтониана (2), соответствующие волновым функциям (4), такой поправки не имеют и равны $\frac{\hbar^{2} k^{2}}{2 m} \pm J$. Во-вторых, стоит отметить, что вычисленный на решении (4) спин, кроме компоненты вдоль мгновенного значения намагниченности, имеет неадиабатическую поправку, линейную по $\beta$.

Волновые функции и спектр в нормальном металле имеют вид

$$
\begin{gathered}
\psi_{+}^{N}=\exp \left(-i \frac{\varepsilon}{\hbar} t+i \mathbf{k r}\right)\left(\begin{array}{l}
1 \\
0
\end{array}\right), \\
\psi_{-}^{N}=\exp \left(-i \frac{\varepsilon}{\hbar} t+i \mathbf{k r}\right)\left(\begin{array}{l}
0 \\
1
\end{array}\right), \\
\varepsilon=\frac{\hbar^{2} \mathbf{k}^{2}}{2 m_{e}} .
\end{gathered}
$$

Эти волновые функции сшиваются с помощью граничных условий (непрерывность $\psi$ и $\psi_{n}^{\prime}$ ) с волновыми функциями (4), после чего необходимо произвести нормировку.

Рассмотрим более детально процедуру решения для электрона, находящегося в состоянии $\left(\begin{array}{l}1 \\ 0\end{array}\right)$, падающего на границу. Волновая функция в нормальном металле имеет вид

$$
\begin{aligned}
\Psi_{R+}= & \xi_{+}\left(e^{-i \frac{\varepsilon}{\hbar} t-i k x}\left(\begin{array}{l}
1 \\
0
\end{array}\right)+A e^{-i \frac{\varepsilon}{\hbar} t+i k x}\left(\begin{array}{l}
1 \\
0
\end{array}\right)\right. \\
& \left.+B e^{-i \frac{\varepsilon}{\hbar} t+i k^{\prime} x}\left(\begin{array}{l}
0 \\
1
\end{array}\right)\right),
\end{aligned}
$$

где поперечный квазиимпульс опущен; $\xi_{+}-$нормировочная константа, $A, B-$ коэффициенты отражения от границы с сохранением и изменением спина. Важно здесь заметить, что энергия электрона после спин-флипа $\varepsilon^{\prime}$ отличается от энергии падающего электрона $\varepsilon$. Ось $x$ перпендикулярна границе металла и ферромагнетика, волновые числа в этом направлении определяются энергией и равны $k=\sqrt{\frac{2 m_{e}}{\hbar^{2}}} \varepsilon, k^{\prime}=\sqrt{\frac{2 m_{e}}{\hbar^{2}} \varepsilon^{\prime}}$. Электрон в ферромагнетике имеет волновую функцию

$$
\psi_{L+}=\xi_{+}\left(C \psi_{+}^{F}\left(\varepsilon_{1}, k_{1}\right)+D \psi_{-}^{F}\left(\varepsilon_{2}, k_{2}\right)\right),
$$

где волновые функции $\psi_{ \pm}^{F}$ определяются (4), $C, D-$ амплитуды прохождения, $\varepsilon_{1}=\varepsilon-\frac{\hbar \Omega}{2}$ определяется из условия одинаковых временны́х зависимостей первой 
компоненты спинора падающей и прошедшей волны (следующего из граничных условий), $\varepsilon_{2}=\varepsilon-\frac{\hbar \Omega}{2}$ определяется из условия одинаковых временных зависимостей для второй компоненты спинора обеих „прошедших“ волн; $k_{1,2}=-i \kappa_{1,2}$ определяются из (5) как

$$
\kappa_{1,2}=\sqrt{\frac{2 m_{e}}{\hbar^{2}}\left(U-\varepsilon \pm J\left(1-\beta \sqrt{1-m^{2}} \pm \beta\right)\right)} .
$$

Поскольку мы рассматриваем ферромагнитный диэлектрик, эти волновые числа мнимые и происходит полное отражение электронов от границы. Тем не менее, процессы спин-флипа напрямую связаны с проникновением волновых функций электронов в диэлектрик и пропадают, если потенциал $U$ стремится к бесконечности.

Из граничных условий определяется, во-первых, энергия электрона, отраженного с переворотом спина

$$
\varepsilon^{\prime}=\varepsilon-\hbar \Omega \text {. }
$$

Во-вторых, определяются коэффициенты отражения

$A=-\frac{a_{+} b_{-}\left(\kappa_{2}-i k^{\prime}\right)\left(\kappa_{1}+i k\right)-a_{-} b_{+}\left(\kappa_{1}-i k^{\prime}\right)\left(\kappa_{2}+i k\right)}{a_{+} b_{-}\left(\kappa_{2}-i k^{\prime}\right)\left(\kappa_{1}-i k\right)-a_{-} b_{+}\left(\kappa_{1}-i k^{\prime}\right)\left(\kappa_{2}+i k\right)}$,

$$
B=\frac{2 i k b_{+} b_{0}\left(\kappa_{1}-\kappa_{2}\right)}{a_{+} b_{-}\left(\kappa_{2}-i k^{\prime}\right)\left(\kappa_{1}-i k\right)-a_{-} b_{+}\left(\kappa_{1}-i k^{\prime}\right)\left(\kappa_{2}-i k\right)} .
$$

Из этих формул видно, что происходит полное отражение электронов от границы. Переворот спина отсутствует, если параметр адиабатичности $\beta$ равен нулю. Наконец, можно определить нормировочный коэффициент

$$
\xi_{+}=\sqrt{\frac{m_{e}}{(2 \pi)^{3} \hbar^{2} k}} .
$$

Аналогичные вычисления нетрудно выполнить и для второй спиновой поляризации $\left(\begin{array}{l}0 \\ 1\end{array}\right)$ падающего на границу электрона. Коэффициенты отражения без изменения спина $A^{\prime}$ и с изменением спина $B^{\prime}$ здесь имеют вид

$A=-\frac{a_{+} b_{-}\left(\kappa_{2}+i k^{\prime}\right)\left(\kappa_{1}-i k\right)-a_{-} b_{+}\left(\kappa_{1}+i k^{\prime}\right)\left(\kappa_{2}-i k\right)}{a_{+} b_{-}\left(\kappa_{2}-i k^{\prime}\right)\left(\kappa_{1}-i k\right)-a_{-} b_{+}\left(\kappa_{1}-i k^{\prime}\right)\left(\kappa_{2}-i k\right)}$,

$$
B=\frac{-2 i k^{\prime} a_{+} a_{-}\left(\kappa_{1}-\kappa_{2}\right)}{a_{+} b_{-}\left(\kappa_{2}-i k^{\prime}\right)\left(\kappa_{1}-i k\right)-a_{-} b_{+}\left(\kappa_{1}-i k^{\prime}\right)\left(\kappa_{2}-i k\right)} .
$$

а нормировочный коэффициент

$$
\xi_{-}=\sqrt{\frac{m_{e}}{(2 \pi)^{3} \hbar^{2} k^{\prime}}}
$$

Стоит отметить, что для этого случая удобно считать энергию падающего на границу электрона равной $\varepsilon^{\prime}$, а энергию отраженного со спин-флипом электрона $\varepsilon$. При этом сохраняется соотношение (14) между ними и остаются справедливыми приведенные выше формулы для волновых чисел.

На найденных волновых функциях нетрудно найти среднее от квантово-механического оператора потока спина

$$
\hat{J}_{j k}=-i \frac{\hbar^{2}}{4 m_{e}} \nabla_{j} \hat{\sigma}_{k},
$$

после чего необходимо произвести усреднение по всем состояниям электронов. Интегрирование по поперечному квазиимпульсу приводит к появлению плотности состояний

$$
\nu_{ \pm}(\widetilde{\varepsilon})=\frac{2 m_{e}}{\hbar^{2}} \pi\left(\varepsilon_{F}-\widetilde{\varepsilon}+\frac{\hbar \Omega}{2} \mu \frac{\hbar \Omega}{2}\right),
$$

где $\widetilde{\varepsilon}$ соответствует энергии $\varepsilon$ после вычета из нее энергии поперечного движения. Тогда компоненту среднего поток спина через границу $J_{x k}$, просуммированного по двум спиновым состояниям, вдоль оси $z$ (параллельной средней намагниченности) можно записать в виде

$$
\begin{aligned}
J_{x z}(\widetilde{\varepsilon}) & =-\frac{1}{16 \pi^{3}} \\
& \times\left(\left(v_{+}-v_{-}\right)\left(1-|A|^{2}\right)+v_{+} \frac{k^{\prime}}{k}|B|^{2}-v_{-} \frac{k}{k^{\prime}}\left|B^{\prime}\right|^{2}\right),
\end{aligned}
$$

где подразумевается, что $J_{i k}(\widetilde{\varepsilon})$ обозначает плотность спинового тока по энергии, полученную после суммирования по спиновым состояниям и интегрирования по поперечному квазиимпульсу. Для других компонент спинового тока имеем

$$
\begin{aligned}
& J_{x x}(\widetilde{\varepsilon})=\frac{1}{32 \pi^{3}}\left(k+k^{\prime}\right)\left(\frac{A^{*} B v_{+}}{k}+\frac{A^{\prime} B^{\prime *} \nu_{-}}{k^{\prime}}\right) e^{i \Omega t}+c . c ., \\
& J_{x y}(\widetilde{\varepsilon})=\frac{1}{32 \pi^{3}}\left(k+k^{\prime}\right)\left(\frac{A^{*} B \nu_{+}}{k}+\frac{A^{\prime} B^{\prime *} \nu_{-}}{k^{\prime}}\right) e^{i \Omega t}+c . c . .
\end{aligned}
$$

Из (23) видно, что, хотя мы выбрали энергии для двух спиновых состояний таким образом, что между ними имеется симметрия $|A|=\left|A^{\prime}\right|$ (см. (15), (18)) и $\frac{k^{\prime}}{k}|B|^{2}=\frac{k}{k^{\prime}}\left|B^{\prime}\right|^{2}$ (см. (16), (19), а также (6), (7)), возникающая при таком выборе энергий разница в плотности состояний, которая согласно (22) пропорциональна $\hbar \Omega$, приводит для всех состояний спектра к ненулевому спиновому току (23), который также будет пропорционален $\hbar \Omega$. (В принципе, возможен другой выбор энергии для спина $\left(\begin{array}{l}0 \\ 1\end{array}\right)$, такой, что энергия падающего электрона равна $\varepsilon$, а рассеянного с изменением спина $\varepsilon+\hbar \Omega$; в этом случае плотность состояний для двух спиновых подзон будет одинакова, но разными окажутся коэффициенты отражения с сохранением и изменением спина, причем разница будет пропорциональна $\hbar \Omega$.) Аналогичным образом, и в (24), (25) ненулевой результат получается из-за отличия в плотности состояний для двух спиновых подзон. Кроме того, стоит отметить, 
что комплексные амплитуды двух компонент спинового тока, перпендикулярных средней намагниченности (компоненты $x$ и $y$ ) одинаковы по величине и отличаются по фазе на $\frac{\pi}{2}$.

Для получения полного спинового тока выражения (23)-(25) необходимо проинтегрировать по всем энергиям электронов. Поскольку, как сказано выше, для каждого состояния эти выражения линейны по $\hbar \Omega$, можно не следить за величинами такого порядка в пределах и интегрировать от 0 до $\varepsilon_{F}$. Интеграл по энергии берется в приближении $\left(U-\varepsilon_{F}\right) \gg J, \hbar \Omega$. При этом мы оставляем только первый порядок по $\hbar \Omega$ (или по параметру адиабатичности $\beta$ ) и первый неисчезающий порядок по $J$ (или по $\left.\frac{J}{\varepsilon_{F}}\right)$.

\section{3. Результаты и обсуждение}

Обсуждая использованный в настоящей работе подход, важно заметить, что, хотя говорится об отраженных от границы электронах, здесь фактически не рассматривается рассеяние электронов проводимости, присутствующих в металле, на границе с диэлектриком. Вместо этого мы ищем электронные состояния в системе. В случае, когда диэлектрик не является ферромагнитным, в металле имеется двукратное вырождение по спину. Состояние электронов в этом случае устроено таким образом, что и в сторону границы, и в противоположную сторону распространяется электрон с некоторым спином. Граница связывает состояния с противоположным направлением распространения. Говоря об отражении электрона от границы, мы подразумеваем такое связанное состояние. Если диэлектрик ферромагнитный, но осцилляций намагниченности нет, спиновые состояния по-прежнему не смешиваются, однако фаза отраженного электрона может различаться для двух разных значений спина. В случае, когда намагниченность в диэлектрике осциллирует, граница связывает состояния с разным спином. Поток спина при этом возникает, как было сказано выше, из-за разной плотности состояний для двух спиновых подзон (которые уже не являются „чистыми“ по спину). Локальное равновесие между спиновыми подзонами нарушается; оно, однако, восстанавливается за счет спиновой релаксации на расстоянии, равном длине спиновой релаксации. Спиновая релаксация в данной работе не рассматривается; очевидно, что эффект спинового насоса имеет место на расстоянии от границы, меньшем, чем длина релаксации спина. В принципе, можно рассматривать связь состояний, распространяющихся в двух направлениях, за счет границы как процесс рассеяния между двумя электронными резервуарами, в каждом из которых по отдельности выполняется статистика Ферми. Однако такое рассмотрение соответствует расстоянию от границы, большому по сравнению с длиной спиновой релаксации; эффект на таком расстоянии был бы крайне мал. Мы же рассматриваем область, находящуюся в непосредственной близости от границы, находящуюся на расстоянии, меньшем, чем длина релаксации спина. В этой области находятся одноэлектронные состояния с учетом границы (снято вырождение по направлению распространения электронов и, кроме того, смешивается спин) и уже они заполняются в соответствии с распределением Ферми (таким образом, учитывается принцип запрета Паули). Заметим также, что в настоящей работе не рассматриваются переходные процессы, связанные с установлением такого состояния.

В отсутствие осцилляций намагниченности процессов c переворотом спина нет, коэффициент отражения без изменения спина равен по модулю единице. Поэтому потоки спина для каждой из двух спиновых подзон падающих на границу электронов в точности равны нулю. В случае, когда осцилляции намагниченности есть, в нулевом порядке по $\beta$ процессы с переворотом спина для двух падающих на границу спиновых состояний в точности компенсируют друг друга, что соответствует качественным соображениям. В первом порядке по $\beta$ появляется поток спина, связанный с осцилляциями намагниченности. Для текущего по нормали к поверхности (вдоль оси $x$ ) спинового тока получаем в младшем неисчезающем порядке по $\frac{J}{\varepsilon_{F}}$

$$
\begin{aligned}
J_{x z}= & -\frac{1-\sqrt{1-m^{2}}+m^{2}}{8 \pi^{2}} \frac{m_{e}}{\hbar^{2}} \varepsilon_{F}^{2} \frac{\hbar \Omega}{J}\left(\frac{J}{\varepsilon_{F}}\right)^{3} \frac{\varepsilon_{F}}{U} \\
& \times\left(\frac{\varepsilon_{F}}{U}+\ln \left(1-\frac{\varepsilon_{F}}{U}\right)\right), \\
J_{x x}= & \frac{m_{e}}{\hbar^{2}} \frac{i m}{8 \pi^{2}} \varepsilon_{F}^{2} \frac{\hbar \Omega}{J}\left(\frac{J}{\varepsilon_{F}}\right)^{2} \\
& \times\left(\frac{\varepsilon_{F}}{U}+\ln \left(1-\frac{\varepsilon_{F}}{U}\right)\right) e^{i \Omega t}+c . c ., \\
J_{x y}= & \frac{m_{e}}{\hbar^{2}} \frac{m}{8 \pi^{2}} \varepsilon_{F}^{2} \frac{\hbar \Omega}{J}\left(\frac{J}{\varepsilon_{F}}\right){ }^{2} \\
& \times\left(\frac{\varepsilon_{F}}{U}+\ln \left(1-\frac{\varepsilon_{F}}{U}\right)\right) e^{i \Omega t}+c . c . .
\end{aligned}
$$

Видно, что поток спина, параллельного равновесной намагниченности, постоянный, в то время, как спин, перпендикулярный ей, осциллирует на частоте осцилляций намагниченности и, кроме того, вращается (потоки в направлении $x$ и $y$ имеют сдвиг фазы на $\left.\frac{\pi}{2}\right)$.

Заметим, во-первых, что результат (26)-(28) $m=0$ обращается в ноль при (намагниченность постоянна и направлена по $z$ ) и имеет максимум при (вся намагниченность осциллирует, постоянной компоненты намагниченности нет), что соответствует соображениям разумности. Выписанные в (27) и (28) значения осциллирующего спинового тока соответствуют первому слагаемому в феноменологическом выражении (1), а постоянный поток спина (26) — второму. При этом второй много меньше первого, в качестве малого параметра здесь выступает $\frac{J}{U}$. Можно записать для $J_{x x}, J_{x y}$ 
слагаемые того же порядка, что и для постоянного потока спина

$$
\begin{aligned}
\delta J_{x x} & =\frac{m_{e}}{\hbar^{2}} \frac{1-\sqrt{1-m^{2}}}{8 \pi^{2}} \varepsilon_{F}^{2} \frac{\hbar \Omega}{J}\left(\frac{J}{\varepsilon_{F}}\right)^{3}\left(\frac{\varepsilon_{F}}{U}\right) \\
& \times\left(\arcsin \frac{\varepsilon_{F}}{U}-\sqrt{\frac{\varepsilon_{F}}{U}\left(1-\frac{\varepsilon_{F}}{U}\right)}\right) e^{i \Omega t}+c . c ., \\
\delta J_{x y} & =i \frac{m_{e}}{\hbar^{2}} \frac{1-\sqrt{1-m^{2}}}{8 \pi^{2}} \varepsilon_{F}^{2} \frac{\hbar \Omega}{J}\left(\frac{J}{\varepsilon_{F}}\right)^{3}\left(\frac{\varepsilon_{F}}{U}\right) \\
& \times\left(\arcsin \frac{\varepsilon_{F}}{U}-\sqrt{\frac{\varepsilon_{F}}{U}\left(1-\frac{\varepsilon_{F}}{U}\right)}\right) e^{i \Omega t}+c . c . .
\end{aligned}
$$

Эти величины соответствуют феноменологической формуле $J_{i j} \sim\left[\mathbf{M}_{0} \times \frac{d \mathbf{m}}{d t}\right]$ в (1), где $\mathbf{M}_{0}-$ не зависящая от времени компонента намагниченности. Поскольку эта величина равна нулю при $m=1$, когда вся намагниченность осциллирует, (29) и (30) обращаются в ноль как для $m=0$, так и для $m= \pm 1$.

На рис. 1 изображена зависимость эффектов постоянного и переменного спинового насоса от амплитуды осцилляции намагниченности $m$. Как уже отмечалось, постоянный и переменный эффекты младшего порядка, соответствующие (26)-(28), обращаются в ноль при $m=0$ и максимальны при $m= \pm 1$, в то время, как поправка к переменному эффекту, как и должно быть из симметрийных соображений, обращается в нуль для этих значений и имеет максимум модуля при $m= \pm \frac{1}{\sqrt{2}}$. Анализ зависимости эффекта от амплитуды осцилляции намагниченности позволит получить частотную зависимость эффекта спинового насоса при известной частотной зависимости амплитуды осцилляций М вблизи частоты ферромагнитного резонанса.

Для анализа зависимости эффекта спинового насоса от потенциала диэлектрика полезно выписать асимпто-

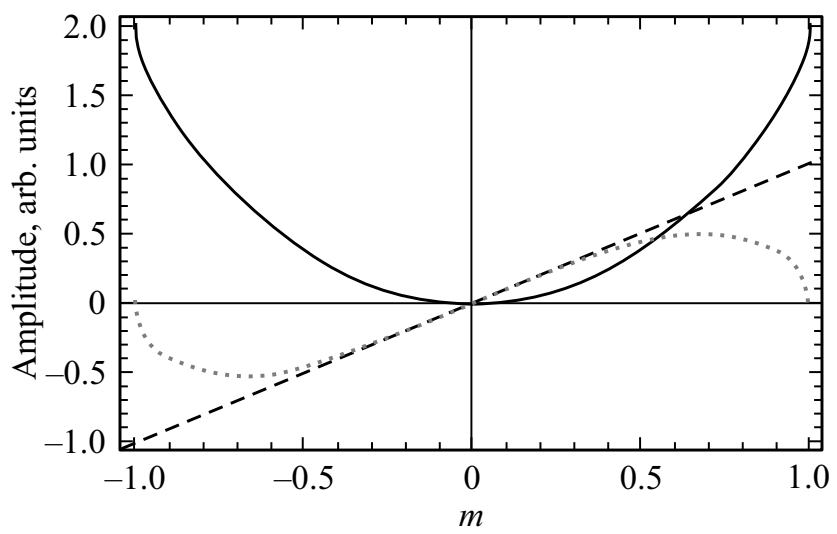

Рис. 1. Зависимость амплитуды постоянного эффекта спинового насоса (сплошная линия), переменного эффекта младшего по $\frac{J}{U}$ порядка (штриховая линия) и следующего порядка, совпадающего с порядком постоянного эффекта (пунктирная линия) от амплитуды осцилляции намагниченности $m$.

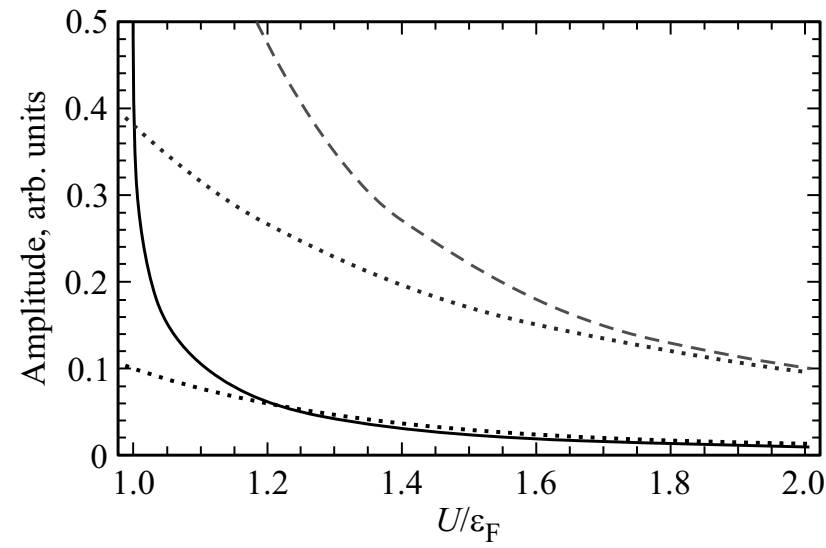

Рис. 2. Характерная зависимость постоянного (сплошная линия) и переменного (штриховая линия) эффекта от потенциального барьера $U$, нормированного на энергию Ферми, для $\frac{J}{\varepsilon_{F}}=0.2, m=0.5$. Пунктиром показаны асимптоты при $U \gg \varepsilon_{F}$.

тическую формулу для ответа $(26)-(28)$ в приближении $U \gg \varepsilon_{F}$

$$
\begin{gathered}
J_{x z}=\frac{1-\sqrt{1-m^{2}}+m^{2}}{8 \pi^{2}} \frac{m_{e}}{\hbar^{2}} \varepsilon_{F}^{2} \frac{\hbar \Omega}{J}\left(\frac{J}{\varepsilon_{F}}\right)^{3}\left(\frac{\varepsilon_{F}}{U}\right)^{3}, \\
J_{x x}=-\frac{m_{e}}{\hbar^{2}} \frac{i m}{8 \pi^{2}} \varepsilon_{F}^{2} \frac{\hbar \Omega}{J}\left(\frac{J}{\varepsilon_{F}}\right)^{3}\left(\frac{\varepsilon_{F}}{U}\right)^{2} e^{i \Omega t}+c . c ., \\
J_{x y}=\frac{m_{e}}{\hbar^{2}} \frac{m}{8 \pi^{2}} \varepsilon_{F}^{2} \frac{\hbar \Omega}{J}\left(\frac{J}{\varepsilon_{F}}\right)^{3}\left(\frac{\varepsilon_{F}}{U}\right)^{2} e^{i \Omega t}+c . c . .
\end{gathered}
$$

На рис. 2 изображены зависимости величины эффекта спинового насоса от потенциала $U$ для $\frac{J}{\varepsilon_{F}}=0.2, m=0.5$. Асимптоты (31)-(33) показаны пунктиром. Как и следовало ожидать, с ростом $U$ величина обоих эффектов падает. Это объясняется тем, что глубина проникновения волновых функций электронов в ферромагнитный изолятор уменьшается с ростом $U$.

Интересно также сравнить члены младшего (27), (28) и следующего (29), (30) по $\frac{J}{\varepsilon_{F}}$ порядков в выражении, полученном для переменного эффекта. Асимптотическое выражение для (29), (30) при $u \gg \varepsilon_{F}$ имеет вид

$$
\begin{aligned}
\delta J_{x x}= & -\frac{m_{e}}{\hbar^{2}} \frac{m \sqrt{1-m^{2}}}{8 \pi^{2}} \varepsilon_{F}^{2} \\
& \times \frac{\hbar \Omega}{J}\left(\frac{J}{\varepsilon_{F}}\right)^{3}\left(\frac{\varepsilon_{F}}{U}\right)^{3 / 2} e^{i \Omega t}+c . c ., \\
\delta J_{x y}= & -i \frac{m_{e}}{\hbar^{2}} \frac{m \sqrt{1-m^{2}}}{8 \pi^{2}} \varepsilon_{F}^{2} \\
& \times \frac{\hbar \Omega}{J}\left(\frac{J}{\varepsilon_{F}}\right)^{3}\left(\frac{\varepsilon_{F}}{U}\right)^{3 / 2} e^{i \Omega t}+c . c . .
\end{aligned}
$$

На рис. 3 показаны амплитуды двух вкладов в переменный эффект в зависимости от $U$, также для $\frac{J}{\varepsilon_{F}}=0.2$, 


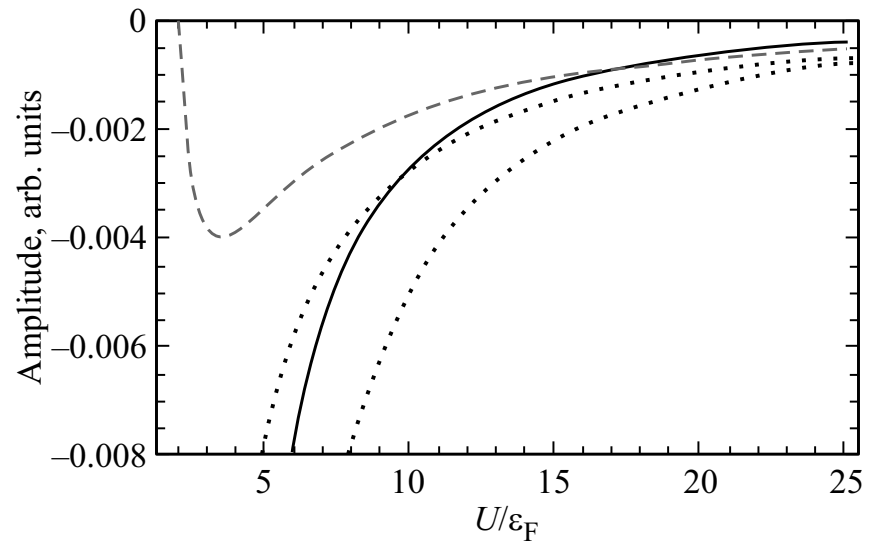

Рис. 3. Характерная зависимость младшего по $\frac{J}{\varepsilon_{F}}$ (сплошная линия) и следующего порядка (штриховая линия) переменного эффекта от потенциального барьера $U$, нормированного на энергию Ферми, для $\frac{J}{\varepsilon_{F}}=0.2, m=0.5$. Пунктиром показаны асимптоты при $U \gg \varepsilon_{F}$.

$m=0.5$. Поскольку асимптотически поправка следующего порядка по $\frac{J}{\varepsilon_{F}}$ спадает при больших $U$ медленнее, чем младший по $\frac{J}{\varepsilon_{F}}$ вклад, эта поправка может быть сравнима с вкладом младшего порядка и даже превзойти его при выполнении условия $U>\alpha \varepsilon_{F}\left(\frac{\varepsilon_{F}}{J}\right)^{2}$, коэффициент $\alpha(m) \sim 1$ зависит от амплитуды осцилляций намагниченности $m$. Из рис. 3 видно, что для выбранных параметров это происходит при $\frac{U}{\varepsilon_{F}} \sim 17$. Для реальных материалов такая ситуация может реализоваться, вероятно, в случае, когда используется плохой проводник, в котором энергия Ферми относительно невелика.

\section{4. Заключение}

Построена простая квантово-механическая модель эффекта спинового насоса при возбуждении ферромагнитного резонанса в магнитном диэлектрике, который имеет плоскую границу с немагнитным металлом. Модель основана на решении уравнения Шредингера для электронов проводимости в металле с последующим усреднением потока спина, который переносят эти электроны, по всем состояниям. Показано, что отражение электронов от изолятора с осциллирующей намагниченностью со спин-флипом приводит к появлению как постоянного, так и переменного (осциллирующего с частотой возбуждающего резонанс СВЧ-поля) потока спина из ферромагнетика в изолятор. При этом эффект постоянного тока мал по сравнению с эффектом переменного тока; в роли малого параметра выступает отношение обменной константы ферромагнетика к величине потенциального барьера между металлом и диэлектриком (больше энергии Ферми). Проанализирована зависимость эффекта от амплитуды осцилляции намагниченности; на основе такого анализа можно получить частотную зависимость эффекта вблизи частоты ферромагнитного резонанса. Также проведен анализ величины постоянного и переменного эффекта от высоты потенциального барьера ферромагнитного диэлектрика. Показано, что оба эффекта стремятся к нулю с ростом барьера, что соответствует представлению о том, что вероятность процессов спин-флипа уменьшается с уменьшением глубины проникновения электронов из нормального металла в ферромагнетик. Кроме того, проанализирован переменный эффект следующего порядка по $\frac{J}{\varepsilon_{F}}$ и показано, что при большой величине потенциального барьера диэлектрика он может доминировать над эффектом младшего порядка по этому параметру.

\section{Финансирование работы}

Работа выполнена при поддержке РФФИ, грант № 18-32-20036.

\section{Список литературы}

[1] Y. Tserkovnyak, A. Brataas, G.E.W. Bauer. Phys. Rev. Lett. 88, 117601 (2002).

[2] A. Brataas, Y. Tserkovnyak, G.E.W. Bauer, B.I. Halperin. Phys. Rev. B 66, 060404(R) (2002).

[3] Y. Tserkovnyak, A. Brataas, G.E.W. Bauer, B.I. Halperin. Rev. Mod. Phys. 77, 1375 (2005).

[4] J. Sinova, S.O. Valenzuela, J. Wunderlich, C.H. Back, T. Jungwirth. Rev. Mod. Phys. 87, 1213 (2015).

[5] E. Saitoh, M. Ueda, H. Miyajima, G. Tatara. Appl. Phys. Lett. 88, 182509 (2006).

[6] H. Wang, C. Du, P.C. Hammel, F. Yang. Appl. Phys. Lett. 104, 202405 (2014).

[7] D. Wei, M. Obstbaum, M. Ribow, C.H. Back, G. Woltersdorf. Nature Commun. 5, 3768 (2014).

[8] G. Tatara, S. Mizukami. Phys. Rev. B 96, 064423 (2017).

[9] С.В. Вонсовский. Магнетизм. Наука, М. (1971).

Редактор Ю.Э. Китаев 